\title{
PERIODIC SOLUTIONS FOR PERTURBED NONLINEAR DIFFERENTIAL EQUATIONS. II
}

\author{
T. G. PROCTOR
}

\begin{abstract}
The existence of periodic solutions of the periodic system $\dot{x}=\varepsilon g(t, x, y, \varepsilon), \dot{y}=f(t, y)+\varepsilon h(t, x, y, \varepsilon)$ is established for small $\varepsilon$ when the solution of the initial value problem $\dot{y}=$ $f(t, y), y(\tau)=\gamma$ is known and some algebraic and smoothness conditions are satisfied.
\end{abstract}

1. Introduction. In this paper we continue the investigation [3] for the existence of periodic solutions for periodically perturbed nonlinear differential equations. Here we assume the unperturbed system has a family of periodic solutions, we study the perturbed system using a generalized variation of constants integral equation and we obtain a set of bifurcation equations. A sufficient condition for the existence of a solution of the bifurcation equations is given and some special relations for these equations in the presence of certain symmetry conditions are developed. The technique is analogous to that developed by Cesari [1] and Hale [2] to study perturbations of linear systems.

2. The unperturbed system. The form of the unperturbed system is adapted from the form used by Hale to study perturbation of linear systems. Let $p$ and $q$ be nonnegative integers, let $|z|=\max _{1 \leqq i \leq k}\left|z_{i}\right|$ for $z$ in $R^{k}$ where $k=p, q$ or $p+q$, and for any positive number $\sigma$ let $S_{p_{\sigma}}$ and $S_{q \sigma}$ denote the closed spheres centered at the origin with radius $\sigma$ in $R^{p}$ and $R^{q}$ respectively. For some $\sigma>0$ let $f(t, y)$ be in $R^{q}$ when $t$ is real and $y$ is in $S_{q \sigma}$ and satisfy

(i) $f$ has period $2 \omega$ in $t$ and is continuous in $t$ and $y$.

(ii) $f$ has continuous first derivatives in $y$.

(iii) There is a $\delta$ in $(0, \sigma)$ so that $\gamma$ in $S_{q \delta}$ implies the solution $\phi(t, \tau, \gamma)$ of

$$
\dot{y}=f(t, y), \quad y(\tau)=\gamma,
$$

exists for $-2 \omega \leqq t-\tau \leqq 2 \omega$ and any number $\tau$.

(iv) $f(t, 0)=0$ for any number $t$.

Received by the editors March 22, 1971.

AMS 1969 subject classifications. Primary 3445, 3453.

Key words and phrases. Periodic solutions, perturbed nonlinear differential equations, variation of constants, contraction mapping. 
(v) There is a nonempty closed set $K \subset S_{\text {q }}$ containing the zero vector so that the function $F$ given by $F(\gamma)=\gamma-\phi(\omega,-\omega, \gamma)$ is invertible on $K$ and that $F^{-1}$ has two continuous derivatives on $F(K)$.

(vi) The elements of the matrix $\Phi(t, \tau, \gamma)=(\partial \phi / \partial \gamma)(t, \tau, \gamma)$ have continuous first and second derivatives in the components of $\gamma$ for $\gamma$ in $S_{a \delta}$ and $-2 \omega \leqq t-\tau \leqq 2 \omega$. In this paper we will study perturbations of the system $\dot{x}=0, \dot{y}=f(t, y),(x, y)$ in $R^{p} \times S_{q \sigma}$. We notice $(x(t), y(t))=(\alpha, 0)$ for $\alpha$ in $R^{p}$ is a periodic solution.

Let $S_{p}$ be the Banach space of continuous functions $x$ from $[-\omega, \omega]$ into $R^{p}$ with

$$
\int_{-\omega}^{\omega} x(t) d t=0, \quad x(-\omega)=x(\omega),
$$

with the supremum norm, and let $L$ be the operator from $\delta_{p}$ into itself given by

$$
L x(t)=\int_{-\omega}^{t} x(s) d s-\frac{1}{2 \omega} \int_{-\omega}^{\omega} \int_{-\omega}^{t} x(s) d s d t .
$$

3. The perturbed system. For $\eta>0$ let $g(t, x, y, \varepsilon)$ and $h(t, x, y, \varepsilon)$ be in $R^{p}$ and $R^{q}$ respectively when $t$ is a real number, $x$ is in $S_{p \sigma}, y$ is in $S_{q \sigma}$ and $0 \leqq \varepsilon \leqq \eta$. Further we suppose $g$ and $h$ have period $2 \omega$ in $t$ and have continuous first and second derivatives in $x, y$ and $\varepsilon$. Our objective is to determine sufficient conditions so that the perturbed system

$$
\begin{aligned}
& \dot{x}=\varepsilon g(t, x, y, \varepsilon), \\
& \dot{y}=f(t, y)+\varepsilon h(t, x, y, \varepsilon),
\end{aligned}
$$

has solutions with period $2 \omega$.

Let $S$ be the Banach space of continuous functions $z$ from $[-\omega, \bar{\omega}]$ into $R^{p+q}$ with $z(-\omega)=z(\omega)$ with the supremum norm, and let $S_{\delta}$ denote the subset of such functions $z$ with $\|z\| \leqq \delta$. For $z$ in $\delta_{\delta}, z(t)=$ $(x(t), y(t))$ in $R^{p} \times R^{q}$, let

$$
M_{z}(\varepsilon)=\frac{1}{2 \omega} \int_{-\omega}^{\omega} g(s, x(s), y(s), \varepsilon) d s,
$$

let $g_{z}$ be the function from $[-\omega, \omega] \times[0, \eta]$ into $R^{p}$ given by

and let

$$
g_{z}(t)=g(t, x(t), y(t), \varepsilon)-M_{z}(\varepsilon),
$$

$$
\eta_{z}(\varepsilon)=\varepsilon \int_{-\omega}^{\omega} \Phi(\omega, s, y(s)) h(s, x(s), y(s), \varepsilon) d s .
$$

THEOREM 1. Let $z$ in $S_{\delta}$ imply $\eta_{z}(\varepsilon)$ is in $F(K)$ for $0 \leqq \varepsilon \leqq \eta$ and let $\alpha$ be in $R^{p}$ with $|\alpha| \leqq \delta / 2$. For $\varepsilon$ sufficiently small there is a unique function $z$ 
in $\mathcal{S}_{\delta}$ such that

$$
\begin{aligned}
& \dot{x}(t)=\varepsilon g(t, x(t), y(t), \varepsilon)-\varepsilon M_{z}(\varepsilon), \\
& \dot{y}(t)=f(t, y(t))+\varepsilon h(t, x(t), y(t), \varepsilon),
\end{aligned}
$$

where $z(t)=(x(t), y(t))$ in $R^{p} \times R^{q}$ and $\int_{-\omega}^{\omega} x(t) d t=2 \omega \alpha$. Further $z$ has continuous first derivatives in $\alpha$ and $\varepsilon$ for $|\alpha| \leqq \delta / 2$ and $\varepsilon$ sufficiently small.

Proof. For $\alpha$ in $R^{p},|\alpha| \leqq \delta / 2$, define the operator $T$ on $S_{\delta}$ by

$$
\begin{aligned}
T z(t) & =\alpha+\varepsilon \operatorname{Lg}_{z}(t), \\
& =\phi\left(t,-\omega, F^{-1}\left(\eta_{z}(\varepsilon)\right)\right)+\int_{-\omega}^{t} \varepsilon \Phi(t, s, y(s)) h(s, x(s), y(s), \varepsilon) d s,
\end{aligned}
$$

where again $z(t)=(x(t), y(t))$. The condition (iv) implies $\phi(t,-\omega, 0)=0$, $-\omega \leqq t \leqq \omega$, and since $F(0)=0$ we have

$$
\lim _{\varepsilon \rightarrow 0} \phi\left(t,-\omega, F^{-1}\left(\eta_{z}(\varepsilon)\right)\right)=0
$$

uniformly for $-\omega \leqq t \leqq \omega$ and $z$ in $S_{\delta}$. Thus we can choose $\varepsilon_{1} \leqq \eta$ so that $0 \leqq \varepsilon \leqq \varepsilon_{1}$ implies $T S_{\delta} \subset S_{\delta}$. A simple calculation shows that for $z_{1}, z_{2}$ in $S_{\delta}$ there is a number $N$ independent of $z_{1}$ and $z_{2}$ such that

$$
\left\|T z_{1}-T z_{2}\right\| \leqq \varepsilon N\left\|z_{1}-z_{2}\right\| ;
$$

consequently we can choose $\varepsilon_{2} \leqq \varepsilon_{1}$ so that $0 \leqq \varepsilon \leqq \varepsilon_{2}$ implies $T$ is a contraction mapping and has a fixed point $z$ which depends on $\alpha$ and $\varepsilon$. Calculation shows that for $z(t)=(x(t), y(t))$, the fixed point, equations (4) hold (see [3]).

The fixed point $z$ is the limit of the sequence

$$
\begin{aligned}
z^{(0)}(t) & =\left(\begin{array}{l}
\alpha \\
0
\end{array}\right), \quad-\omega \leqq t \leqq \omega, \\
z^{(k)} & =T z^{(k-1)}, \quad k=1,2, \cdots,
\end{aligned}
$$

and an easy calculation shows that $\partial z^{(k)} / \partial \alpha, \partial z^{(k)} / \partial \varepsilon$ exists and are continuous for $k=0,1,2, \cdots$. Using the same proof as presented in Hale, pp. 39-40, we see that for $\varepsilon$ sufficiently small, the sequences $\left\{\partial z^{(k)} / \partial \alpha\right\},\left\{\partial z^{(k)} / \partial \varepsilon\right\}$ converges uniformly. This implies $\partial z / \partial \alpha, \partial z / \partial \varepsilon$ exist and are continuous.

COROLlaRY 1. Let the hypothesis of Theorem 1 hold and let $z(t, \alpha, \varepsilon)$ be the function given by the conclusion of Theorem 1 for $-\omega \leqq t \leqq \omega$, $|\alpha| \leqq \delta / 2,0 \leqq \varepsilon \leqq \varepsilon_{0}$. If there is an $\varepsilon_{2} \leqq \varepsilon_{0}$ and a continuous function $\alpha(\varepsilon)$, $0 \leqq \varepsilon \leqq \varepsilon_{2}$ with $|\alpha(\varepsilon)| \leqq \delta / 2$ such that $M_{z}=0$ for $z=z(t, \alpha, \varepsilon)$ then the periodic extension of $z(t, \alpha, \varepsilon)$ is a periodic solution of (2). 
COROLlaRY 2. Let the hypothesis of Theorem 1 hold, let

$$
\frac{1}{2 \omega} \int_{-\omega}^{\omega} g\left(s, \alpha_{0}, 0, \varepsilon\right) d s=0
$$

for some $\alpha_{0}$ in $R^{p},\left|\alpha_{0}\right|<\delta / 2$, and let

$$
\operatorname{det}\left|\frac{\partial}{\partial \alpha} \int_{-\omega}^{\omega} g(s, \alpha, 0, \varepsilon) d s\right| \neq 0
$$

for $\alpha=\alpha_{0}$. There exists $a 0 \leqq \varepsilon_{3} \leqq \varepsilon_{0}$ and a periodic solution $z(t, \varepsilon)$ of (2) for $0 \leqq \varepsilon<\varepsilon_{3}$ with $z(t, 0)=\left(\alpha_{0}, 0\right)$.

The first corollary is clear from Theorem 1 and Corollary 2 follows from the Implicit Function Theorem and Corollary 1.

It is easy to relax the conditions on $f, g, h$ and obtain a function $z(t, \alpha, \varepsilon)$ which satisfies equation (4), say by using the Schauder Fixed Point Theorem. However the dependence of $z$ on $\alpha, \varepsilon$ is not clear and the existence of a solution of $M_{z}=0$ would be in doubt. We will present below some special relations concerning the bifurcation equations $M_{z}=0$ in the presence of symmetry conditions.

4. Symmetry conditions. Let $P$ be a $p \times p$ matrix with $P^{2}=I$, let $Q$ be a $q \times q$ matrix with $Q^{2}=I$ and let $\delta \leqq \sigma /\|P\|, \delta \leqq \sigma /\|Q\|$. Let us further assume the symmetry conditions

$$
\begin{aligned}
P g(-t, P x, Q y, \varepsilon) & =-g(t, x, y, \varepsilon), \\
Q f(-t, Q y) & =-f(t, y), \\
Q h(-t, P x, Q y, \varepsilon) & =-h(t, x, y, \varepsilon),
\end{aligned}
$$

for $-\omega \leqq t \leqq \omega,|x| \leqq \delta,|y| \leqq \delta$, and $0 \leqq \varepsilon \leqq \eta$. Let $X$ be the set of continuous functions $x:[-\omega, \omega] \rightarrow S_{p \delta}$ with $x(-\omega)=x(\omega)$ and $P x(-t)=x(t)$ for $-\omega \leqq t \leqq \omega$. For $x$ in $X$ we notice that Theorem 1 guarantees the existence of a periodic solution of

$$
\dot{y}=f(t, y)+\varepsilon h(t, x(t), y, \varepsilon)
$$

for $\varepsilon$ sufficiently small, say $0 \leqq \varepsilon \leqq \varepsilon_{0}$ if $y$ in $S_{\delta}$ implies $\eta_{y}(\varepsilon)$ is in $F(K)$. Here $p=0$ and $h(t, x, y, \varepsilon)$ is replaced by $\tilde{h}(t, y, \varepsilon)=h(t, x(t), y, \varepsilon)$. We make the further assumption that, for any $x$ in $X$, then $y$ in $\delta_{\delta}$ implies $\eta_{y}(\varepsilon)$ is in $F(K)$. Then $\varepsilon_{0}$ is independent of $x$ and we denote the periodic solution of (6) by $y_{x}$. Further we note by the proof of Theorem 1 that $y_{x}$ is the only periodic solution of (6) lying in $S_{a \delta}$ whose value at $\omega$ is in $K$.

LEMma 1. If $Q K \subset K$ then $Q y_{x}(-t)=y_{x}(t)$. 
Proof. Let $v(t)=Q y_{x}(-t)$, then

$$
\begin{aligned}
\dot{v}(t) & =-Q\left[f\left(-t, y_{x}(-t)\right)+\varepsilon h\left(-t, x(-t), y_{x}(-t), \varepsilon\right)\right] \\
& =f(t, v(t))+\varepsilon h(t, x(t), v(t), \varepsilon),
\end{aligned}
$$

and $v(-\omega)=v(\omega)$. The lemma follows from the uniqueness of $y_{x}$.

Lemma 2. $P \operatorname{Lg}_{z}(-t)=L g_{z}(t)$.

The proof follows from Lemma 1 and a straightforward calculation.

Let $\alpha$ in $R^{p}$ be chosen so that $P \alpha=\alpha,|\alpha| \leqq \delta / 2$, and define a transformation $\mathscr{C}$ from $X$ into the continuous functions $x^{*}:[-\omega, \omega] \rightarrow$ $S_{p \delta}$ with $x^{*}(-\omega)=x^{*}(\omega)$ by

$$
\mathcal{C} x(t)=\alpha+\varepsilon \operatorname{Lg}_{z}(t)
$$

where $z(t)=\left(x(t), y_{x}(t)\right)$.

THEOREM 2. If $Q K \subset K$ then for $\varepsilon$ sufficiently small there is a fixed point $x$ of the operator $\mathcal{C}$ and the function $z(t)=\left(x(t), y_{x}(t)\right)$ ( $x$ is the fixed point) satisfies (4). Further if $P$ is diagonal and if the element in the $j$ column is one then the jth equation in

is zero.

$$
M_{z}(\varepsilon)=\frac{1}{2 \omega} \int_{-\omega}^{\omega} g\left(s, x(s), y_{x}(s), \varepsilon\right) d s
$$

Proof. As in the proof of Theorem 1 for $\varepsilon$ sufficiently small, $\left|y_{x}(t)\right| \leqq$ $\delta,|\mathfrak{C} x(t)| \leqq \delta$ for $-\omega \leqq t \leqq \omega$ and Lemma 2 shows that $\mathscr{C} X \subset X$. To see $\mathfrak{C}$ is continuous let $\Psi$ be all continuous functions $y$ from $[-\omega, \omega]$ into $S_{q \delta}$ with $y(\omega)=y(-\omega)$ and let $U$ be the transformation defined on $X \times \Psi$ into the functions from $[-\omega, \omega]$ to $R^{p}$ given by

$$
\begin{aligned}
U(x, y)(t)= & \phi\left(t,-\omega, F^{-1}\left(\eta_{z}(\varepsilon)\right)\right) \\
& +\int_{-\omega}^{t} \varepsilon \phi(t, s, y(s)) h(x, x(s), y(s), \varepsilon) d s
\end{aligned}
$$

where $z(t)=(x(t), y(t)) . U(, y)$ is continuous uniformly for $y$ in $\Psi$ and we note $U(x):, \Psi \rightarrow \Psi$ is a contraction with fixed point $y_{x}$. Thus for fixed $x$ and $X$ and a sequence $\left\{x_{n}\right\}$ from $X$ with $x_{n} \rightarrow x$ we have

$$
\begin{aligned}
\left\|y_{x_{n}}-U\left(x, y_{x_{n}}\right)\right\| & \leqq\left\|y_{x_{n}}-U\left(x_{n}, y_{x_{n}}\right)\right\|+\left\|U\left(x_{n}, y_{x_{n}}\right)-U\left(x, y_{x_{n}}\right)\right\| \\
& =\left\|U\left(x_{n}, y_{x_{n}}\right)-U\left(x, y_{x_{n}}\right)\right\| \rightarrow 0
\end{aligned}
$$

as $n \rightarrow \infty$. Therefore $y_{x}$ is continuous in $x$ and easy calculations show that $\mathfrak{C}$ is continuous and $\mathscr{C} X$ has compact closure.

By the Schauder Fixed Point Theorem we have the first conclusion. 
The last conclusion follows from $P M_{z}=-M_{z}$ for $z(t)=\left(x(t), y_{x}(t)\right)$, $x$ in $X$.

COROLlaRY 3. Let the hypothesis of Theorem 2 hold with $P=I$. Then there is a p-parameter family of periodic solutions of (2) for $\varepsilon$ sufficiently small.

\section{REFERENCES}

1. L. Cesari, Asymptotic behavior and stability problems in ordinary differential equations, Ergebnisse der Mathematik und ihrer Grenzgebiete, N.F., Heft 16, SpringerVerlag, Berlin, 1959. MR 22 \#9673.

2. J. K. Hale, Oscillations in nonlinear systems, McGraw-Hill, New York, 1963. MR 27 \#401.

3. T. G. Proctor, Periodic solutions for perturbed nonlinear differential equations, Proc. Amer. Math. Soc. 24 (1970), 815-819. MR 41 \#581.

Department of Mathematics, Clemson University, Clemson, South Carolina 29631 